\title{
Viabilidade da utilização de queijo tipo ricota na elaboração de pão de queijo
}

\author{
Viability of using ricotta in preparation of cheese bread
}

\author{
Patrícia Aparecida Pimenta Pereira ${ }^{I^{*}}$ Thaís de Melo Ramos ${ }^{I}$ Adriano Alvarenga Gajo \\ Ulisses Júnior Gomes ${ }^{\text {II }}$
}

\section{RESUMO}

O pão de queijo é um produto genuinamente brasileiro, surgido em época indefinida nas fazendas de Minas Gerais, tendo como matéria-prima básica o polvilho azedo ou doce. Atualmente vem se destacando pelo consumo e pela produção nacional, chegando aos dias atuais até mesmo ao mercado internacional. Apesar de sua aceitação, o pão de queijo ainda não possui um padrão de qualidade e tecnologia de produção definidos devido à grande variedade de ingredientes opcionais. Diversas formulações são comercializadas e identificadas como "pão de queijo". $O$ objetivo deste trabalho foi analisar a viabilidade da utilização de queijo tipo ricota na elaboração de pão de queijo. Foram realizadas análises físicas de densidade, espessura da crosta, coeficiente de expansão, cor, textura e análise sensorial. As porcentagens de queijo utilizadas nas formulações foi $30 \%$ para o queijo meia cura e 30, 40 e 50\% para o queijo tipo ricota, em relação à porcentagem de polvilho azedo. Em relação aos parâmetros analisados, conclui-se que o aumento da porcentagem de queijo tipo ricota na elaboração de pão de queijo tem como consequência um produto mais macio, com menor gomosidade, fracturabilidade, mastigabilidade e com uma espessura da crosta menor, não diferindo sensorialmente de pães de queijo elaborados com queijo minas meia cura. Conclui-se também que é viável produzir pão de queijo com a substituição total do queijo minas meia cura pelo queijo tipo ricota.

Palavras-chave: ricota, pão de queijo, análises fisicas, análise sensorial.

\section{ABSTRACT \\ The cheese bread is a genuinely Brazilian product, which appeared in undefined time in the farms of Minas Gerais,}

\begin{abstract}
with the basic raw material, cassava starch or sour cassava starch. Today has been highlighted by consumption and domestic production, up to today, even to the international market. Despite its acceptance, the cheese bread does not have a standard of quality and production technology defined by the great variety of optional ingredients. Several formulations are marketed and labeled as "cheese bread". The aim of this study was to analyze the feasibility of using ricotta cheese in the preparation of cheese. Physical analyzes density, crustal thickness, coefficient of expansion, color, texture and sensory. The percentages of cheese used in the formulations was $30 \%$ for half the cheese curing and 30\%, 40\% and 50\% for the ricotta cheese, in relation to the percentage of cassava starch. The parameters analyzed it is concluded that increasing the percentage of ricotta cheese in the preparation of cheese bread has resulted in a softer, less gumminess, fracture, and chewiness of the crust with a thickness less, no difference in sensory cheese bread made with cheese half cure. It also follows that it is feasible to produce cheese bread with total replacement of the cheese half cure for ricotta cheese.
\end{abstract}

Key words: ricotta, cheese bread, physicals analysis, sensory analysis.

\section{INTRODUÇÃO}

O pão de queijo é um produto tradicional de Minas Gerais que possui grande aceitação no mercado interno e tendência acentuada de expansão no mercado externo (MINIM et al., 2000) e que pode ser definido como um produto resultante do processo de assar uma massa constituída basicamente de polvilho, queijo,

'Departamento de Ciência dos Alimentos, Universidade Federal de Lavras (UFLA), 37200-000, Lavras, MG, Brasil. E-mail: pattyap2001@yahoo.com.br. *Autor para correspondência.

IIDepartamento de Ciências Exatas, UFLA, Lavras, MG, Brasil. 
água, gordura e sal, podendo conter outros ingredientes, como ovos e leite. Porém, sob a denominação de pão de queijo, podem ser encontrados no mercado diferentes tipos de produtos com características distintas. Essa variação no produto pode ser justificada pela inexistência de padrões de identidade e qualidade (SILVA, 2005). Diversas formulações são comercializadas e igualmente identificadas como "pão de queijo". No mercado, o produto pode ser apresentado já modelado e congelado, em pó para posterior adição de ingredientes ou ainda na forma de massa congelada (MINIM et al., 2000).

De acordo com a Associação Brasileira de Produtores de Pão de Queijo (ABPQ), para se ter ideia do tamanho desse mercado, estima-se que existem atualmente 500 indústrias de pão de queijo no Brasil, a maior parte (70\%) no Estado de Minas Gerais. Entre empresas legalmente registradas e fabricantes informais, a previsão de produção média é de seis mil toneladas mensais (TOMICH et al., 2005).

O pão de queijo pode ser uma fonte reconhecida de carboidratos, além de ser um produto de panificação isento de glúten, o que o coloca como alimento alternativo para pacientes celíacos, alérgicos às proteínas do trigo (PEREIRA et al., 2004).

A doença celíaca se caracteriza como uma enteropatia imuno-mediada e ocorre pela ingestão do glúten em indivíduos geneticamente susceptíveis (TROCONE et al., 2004). A porção antigênica da proteína do glúten para os pacientes celíacos é predominantemente a gliadina do trigo, que apresenta alto teor dos aminoácidos glutamina e prolina (BAPTISTA, 2006).

Considerando que cada vez mais a população está em busca de alimentos de fácil e rápido preparo, como os congelados, o pão de queijo apresenta vantagens de incluir ingredientes de fácil aquisição em sua formulação e sua massa poder ser congelada, satisfazendo as necessidades dos consumidores. Com a possibilidade de congelamento da massa e a grande comercialização das misturas prontas, o pão de queijo ocupa o segundo lugar entre os produtos nacionais mais requisitados pelos brasileiros que vivem no exterior (PEREIRA et al., 2004).

Os queijos mais utilizados na sua produção são a mussarela, parmesão, minas curado e minas padrão (MACHADO, 2003). Atualmente, com a tendência de serem consumidos produtos com menos calorias, a substituição parcial ou total de queijos usualmente utilizados por queijo tipo ricota pode representar uma alternativa interessante, já que esse tipo de queijo apresenta um percentual calórico mais baixo, quando comparado com o queijo meia cura, por ser um produto fabricado a partir do soro do leite.

Utilizando a tabela nutricional proposta por FRANCO (2005), o pão de queijo elaborado com queijo minas meia cura tem em média $60 \mathrm{kcal}$, já o produto produzido com ricota teria $50 \mathrm{kcal}$, representando uma redução calórica de $16,67 \%$. O consumo de produtos com baixas calorias é uma tendência atual, justificando a realização desta pesquisa. Além disso, esse produto poderia favorecer o consumo de proteínas ausentes em outros queijos, como a albumina, e utilizar a ricota, que é um item altamente perecível, em um produto de panificação.

Este trabalho teve como objetivo avaliar a influência da substituição do queijo minas meia cura por queijo tipo ricota sobre as propriedades físicas e sensoriais do pão de queijo, em comparação com a amostra de referência (produzida com queijo meia cura).

\section{MATERIAL E MÉTODOS}

Para a elaboração dos pães de queijo, foram utilizados como matéria-prima: polvilho azedo, queijo minas meia cura, queijo tipo ricota, ovos, leite pasteurizado integral, óleo vegetal e sal, adquiridos em mercado local.

As percentagens dos ingredientes utilizados em relação ao polvilho azedo foram: $100 \%$ de polvilho azedo, $25 \%$ de ovo, $40 \%$ de leite, $25 \%$ de óleo e $4 \%$ de sal.

Foram realizados testes preliminares de formulações de pães de queijo, a fim de obter a melhor formulação mediante avaliação da textura da massa, aparência global e volume dos pães de queijo. Foram utilizadas quatro formulações para a elaboração dos pães de queijo ( 1 - 30\% de queijo meia cura; $2-30 \%$ de queijo tipo ricota; $3-40 \%$ de queijo tipo ricota; e $4-50 \%$ de queijo tipo ricota). Somente a percentagem de queijo utilizado variou em relação à porcentagem de polvilho azedo empregada e, para cada experimento, foram realizadas três repetições.

A produção dos pães seguiu a metodologia descrita por (CLARETO, 2000). Os pães de queijo foram misturados em batedeira planetária durante cinco minutos, em velocidade baixa. A massa foi dividida em porções de $15 \pm 0,5 \mathrm{~g}$ e moldadas manualmente em formato esférico. As unidades de pães de queijo foram assadas em condições padronizadas de tempo (30 minutos) e temperatura $\left(180^{\circ} \mathrm{C}\right)$ em forno elétrico.

Foram realizadas análises de densidade (pela fórmula: densidade $=$ massa/volume. $\mathrm{O}$ volume foi medido pelo método de deslocamento de sementes de painço), espessura da crosta (tanto superior, como 
inferior, medida com paquímetro em quatro pontos diferentes, posteriormente, a média aritmética simples dessas determinações) e coeficiente de expansão pela fórmula: Coeficiente de expansão = (volume do pão de queijo - volume da massa crua)/volume da massa crua * 100, conforme metodologia citada por CLARETO (2000).

Foram realizadas análises de cor para as amostras pão de queijo. Os valores $\mathrm{L}^{*}, \mathrm{a}^{*} \mathrm{e} \mathrm{b}^{*}$ foram determinados, em colorímetro Minolta CR 400, trabalhando com D65 (luz do dia) e utilizando-se os padrões CIElab, em que $\mathrm{L}^{*}=$ mede a luminosidade e varia de $100(\mathrm{cem})$ para superfícies perfeitamente brancas até 0 (zero) para o preto; $a^{*}=$ mede a intensidade de vermelho $(+)$ e verde $(-)$; e b* b $^{*}$ mede a intensidade de amarelo (+) e azul (-).

Para as análises de textura (TPA), foi utilizado o texturômetro Stable Micro Systems (Modelo TA - XT2i), com velocidade de pré-teste de $5,0 \mathrm{~mm} \mathrm{~s}^{-1}$, velocidade de teste de $2,0 \mathrm{~mm} \mathrm{~s}^{-1}$ e velocidade de pós teste de $2,0 \mathrm{~mm} \mathrm{~s}^{-1}$, com distância de compressão de $10,0 \mathrm{~mm}$ e probe de $6 \mathrm{~mm}$ em pães de queijo inteiros. Foram utilizadas seis unidades de pão de queijo para cada tratamento.

Para verificar a influência da utilização de ricota na elaboração de pão de queijo, foi realizada uma análise sensorial com 32 potenciais consumidores, pertencentes à comunidade da Universidade Federal de Lavras (estudantes, professores e funcionários) mediante um teste de aceitação, com a utilização de uma escala hedônica estruturada por nove pontos ( $1=$ desgostei extremamente a $9=$ gostei extremamente). As amostras foram servidas nas seguintes condições: em cabines individuais, em pratos de papel, codificados com algarismos de três dígitos retirados de uma tabela de números aleatórios, em ordem balanceada de apresentação, conforme DELLA MODESTA (1994).

Os dados foram avaliados por análise de variância (ANOVA), seguida de teste de Tukey, a 5\% de significância, para identificar as diferenças em casos significativos. As análises de variância e o teste de médias foram realizados no software Sisvar (FERREIRA, 2003).

\section{RESULTADOS E DISCUSSÃO}

Face aos resultados das características físicas dos pães de queijo elaborados com queijo minas meia cura ou com ricota (Tabela 1), estes apresentaram diferença significativa $(\mathrm{P}<0,05)$ nos atributos de espessura da crosta superior e para o coeficiente de expansão.

Não houve diferença significativa entre as formulações 1 ( $30 \%$ de queijo meia cura) e 4 ( $50 \%$ de ricota) e entre as formulações 2 (30\% de ricota) e 3 ( $40 \%$ de ricota) para a espessura da crosta superior. As formulações com maior quantidade de ricota apresentam, consequentemente, maior teor de lipídeos no sistema, uma vez que a legislação vigente permite adicionar leite até $20 \%$ do volume do soro (CERESER, 2006). Essa quantidade de lipídeo propicia uma massa mais fina e uniforme (MINIM, 2000).

$\mathrm{Na}$ análise do coeficiente de expansão (Tabela 1), pode-se notar que não houve diferença significativa entre as formulações 2 ( $30 \%$ de ricota) e 4 ( $50 \%$ de ricota), com maiores resultados em relação às formulações 1 e 3 (iguais estatisticamente). Isso pode ter acorrido devido à falha no tempo de assamento, uma vez que é incoerente afirmar que um produto com $30 \%$ de queijo meia cura é igual ao produto de $30 \%$ de queijo tipo ricota, mas diferente daquele com $20 \%$ de queijo tipo ricota.

Em relação à cor (Tabela 1), não houve diferença significativa entre as amostras. Isso porque, no tratamento 1 , o polvilho influenciou a cor, e os outros tratamentos foram influenciados também pela quantidade de ricota adicionada. A cor é um dos principais atributos que afetam o consumo por meio da

Tabela 1 - Médias \pm desvio padrão dos resultados obtidos nas análises das características físicas dos pães de queijo elaborados com queijo minas meia cura ou com ricota.

\begin{tabular}{|c|c|c|c|c|c|c|c|}
\hline \multirow{2}{*}{ Formulações } & \multirow{2}{*}{ ECS (mm) } & \multirow{2}{*}{$\mathrm{ECI}(\mathrm{mm})$} & \multirow{2}{*}{$\mathrm{D}\left(\mathrm{g} \mathrm{cm}^{-3}\right)$} & \multicolumn{3}{|c|}{ 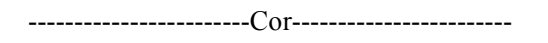 } & \multirow{2}{*}{ CE $(\%)$} \\
\hline & & & & $\mathrm{L}$ & $\mathrm{a}$ & $\mathrm{b}$ & \\
\hline 1 & $2,21 \mathrm{~b} \pm 0,43$ & $1,79 \mathrm{a} \pm 1,54$ & $0,28 \mathrm{a} \pm 0,21$ & $77,83 \mathrm{a} \pm 0,14$ & $2,24 \mathrm{a} \pm 0,29$ & $26,81 \mathrm{a} \pm 1,10$ & $187,34 \mathrm{~b} \pm 0,85$ \\
\hline 2 & 3,33 a $\pm 1,03$ & $2,67 \mathrm{a} \pm 2,34$ & $0,26 \mathrm{a} \pm 0,32$ & $77,61 \mathrm{a} \pm 1,18$ & $2,23 \mathrm{a} \pm 0,41$ & $21,54 \mathrm{a} \pm 0,26$ & $288,36 \mathrm{a} \pm 1,10$ \\
\hline 3 & $3,08 \mathrm{a} \pm 0,32$ & $2,37 \mathrm{a} \pm 1,23$ & $0,29 \mathrm{a} \pm 0,45$ & $75,98 \mathrm{a} \pm 0,68$ & $3,50 \mathrm{a} \pm 1,36$ & $25,11 \mathrm{a} \pm 1,20$ & $205,98 b \pm 0,78$ \\
\hline 4 & $1,50 \mathrm{~b} \pm 0,66$ & $1,42 \mathrm{a} \pm 0,44$ & $0,22 \mathrm{a} \pm 1,56$ & $82,50 \mathrm{a} \pm 2,67$ & $1,07 \mathrm{a} \pm 3,46$ & $21,79 \mathrm{a} \pm 0,65$ & $317,78 \mathrm{a} \pm 0,54$ \\
\hline
\end{tabular}

Médias seguidas da mesma letra, nas colunas, não diferem estatisticamente entre si pelo Teste de Tukey, a $5 \%$ de probabilidade. ECS- espessura da crosta superior; ECI- espessura da crosta inferior; D- densidade; CE- Coeficiente de expansão. 
percepção da qualidade do produto, podendo até ser usada como estimativa dos componentes nutricionais para índices de qualidade, influenciando também a tomada de decisão de compra pelo consumidor (FIATES et al., 2008).

Não houve diferença significativa para densidade dos pães de queijo. Isso porque todos os ingredientes utilizados foram em proporção ao conteúdo de polvilho azedo, fazendo com que a densidade fosse a mesma (CERESER, 2006). Já o aumento da percentagem de utilização da ricota fez com que a espessura da crosta superior diminuísse, sendo obtido um produto com crosta mais fina, em relação à formulação com $30 \%$ de queijo minas meia cura (1). Isso se deve ao alto teor de umidade da ricota (RIBEIRO et al., 2005).

A análise do Perfil de Textura (TPA) instrumental aplica sucessivas forças deformantes, numa simulação da ação de compressão e do corte dos dentes durante a mastigação (LAU et al., 2000). Os dados contidos na tabela 2 indicam que a formulação 4 apresentou menor dureza, fracturabilidade, adesividade, gomosidade e mastigabilidade em relação às outras formulações e isso se deve ao maior teor de ricota.

$\mathrm{O}$ aumento do teor de ricota faz com que a massa fique com uma quantidade de água maior e isso gera um produto mais macio e de melhor mastigabilidade, uma vez que o queijo contribui para o aroma e o sabor típicos dos produtos, complementando a estruturação do miolo do pão de queijo e auxiliando na obtenção de melhor textura do produto final. Esses aspectos contribuem para a elasticidade e melhor aspecto da casca, além de conferir uma maior maciez e uniformidade às células do miolo.

Os aspectos de elasticidade e resistência não apresentaram diferença significativa entre si, possibilitando averiguar que os tratamentos aplicados não influenciaram esses atributos.

Os resultados das médias obtidas pela análise sensorial estão contidos na tabela 3 , observando-se que, em todas as formulações, não houve diferença significativa.

$\mathrm{Na}$ análise dos dados de textura (Tabela 2) com os da análise sensorial (Tabela 3), observa-se que a formulação 4, com a maior aceitação, apresentou-se com as menores médias de textura, evidenciando que os provadores preferiram um pão de queijo mais macio.

\section{CONCLUSÃO}

Em relação aos parâmetros analisados, conclui-se que o aumento da porcentagem de ricota na elaboração de pão de queijo tem como consequência um produto mais macio, com menor gomosidade, fracturabilidade, mastigabilidade e uma espessura da crosta menor, não diferindo sensorialmente de pães de queijo elaborados com queijo minas meia cura, de modo que é viável produzir pão de queijo com a substituição total do queijo minas meio cura pela ricota.

Tabela 2 - Valores médios \pm desvio padrão dos parâmetros analisados pelo perfil de textura (TPA) dos pães de queijo elaborados com queijo minas meia cura ou com ricota.

\begin{tabular}{cllcccccc}
\hline Formulações & DU $(\mathrm{g})$ & FRA $(\mathrm{g})$ & ADE $\left(\mathrm{g}^{*} \mathrm{~s}\right)$ & ELA & COE & GOM & MAS & RES \\
\hline 1 & $2064,67 \mathrm{a}$ & $2077,02 \mathrm{a}$ & $-0,51 \mathrm{a}$ & $0,80 \mathrm{a}$ & $0,36 \mathrm{~b}$ & $733,16 \mathrm{a}$ & $587,57 \mathrm{a}$ & $0,16 \mathrm{a}$ \\
& $\pm 0,28$ & $\pm 1,30$ & $\pm 1,54$ & $\pm 0,43$ & $\pm 0,23$ & $\pm 0,98$ & $\pm 0,34$ & $\pm 0,98$ \\
2 & $1604,43 \mathrm{~b}$ & $1388,08 \mathrm{~b}$ & $-0,39 \mathrm{a}$ & $0,82 \mathrm{a}$ & $0,39 \mathrm{~b}$ & $620,61 \mathrm{a}$ & $508,75 \mathrm{a}$ & $0,16 \mathrm{a}$ \\
& $\pm 0,45$ & $\pm 0,21$ & $\pm 0,29$ & $\pm 0,34$ & $\pm 0,34$ & $\pm 2,01$ & $\pm 0,43$ & $\pm 0,21$ \\
3 & $1606,90 \mathrm{~b}$ & $961,88 \mathrm{c}$ & $-0,18 \mathrm{~b}$ & $0,78 \mathrm{a}$ & $0,39 \mathrm{~b}$ & $635,34 \mathrm{a}$ & $494,70 \mathrm{a}$ & $0,15 \mathrm{a}$ \\
& $\pm 1,14$ & $\pm 2,19$ & $\pm 0,67$ & $\pm 0,77$ & $\pm 0,32$ & $\pm 0,53$ & $\pm 2,65$ & $\pm 0,45$ \\
4 & $612,19 \mathrm{c}$ & $122,30 \mathrm{~d}$ & $-0,01 \mathrm{~b}$ & $0,84 \mathrm{a}$ & $0,53 \mathrm{a}$ & $326,54 \mathrm{~b}$ & $275,62 \mathrm{~b}$ & $0,22 \mathrm{a}$ \\
& $\pm 0,31$ & $\pm 0,54$ & $\pm 1,09$ & $\pm 0,65$ & $\pm 0,45$ & $\pm 0,22$ & $\pm 0,77$ & $\pm 0,76$ \\
\hline
\end{tabular}

DU- Dureza

GOM- Gomosidade

FRA- Fracturabilidade

MAS- Mastigabilidade

ADE- Adesividade

RES- Resistência

ELA- Elasticidade

COE- Coesividade

Médias seguidas da mesma letra, nas colunas, não diferem estatisticamente entre si pelo Teste de Tukey, a 5\% de probabilidade. 
Tabela 3 - Médias \pm desvio padrão dos escores para as formulações.

\begin{tabular}{cc}
\hline Formulações & Aceitação \\
\hline 1 & $6,91 \mathrm{a} \pm 0,45$ \\
2 & $6,35 \mathrm{a} \pm 0,98$ \\
3 & $6,84 \mathrm{a} \pm 0,56$ \\
4 & $7,25 \mathrm{a} \pm 0,32$ \\
\hline
\end{tabular}

Médias seguidas da mesma letra, nas colunas, não diferem estatisticamente entre si pelo Teste de Tukey, a 5\% de probabilidade.

\section{REFERÊNCIAS}

BAPTISTA, M. L. Doença celíaca: uma visão contemporânea. Pediatria, São Paulo, v.28, n.4, p.262-271, 2006. Disponível em: <http://www.pediatriasaopaulo.usp.br/upload/pdf/1189.pdf $>$. Acesso em: 04 set. 2010.

CERESER, N. D. Pesquisa de Listeria monocytogenes, Salmonella spp, Staphylococcus aureus e indicadores higiênico-sanitários em ricota de produção industrial. 2006. 62f. Dissertação (Mestrado em Medicina Veterinária) Curso de Pós-graduação em Ciências Agrárias e Veterinária, Universidade Estadual Paulista.

CLARETO, S. S. Influência da formulação e da adição de substitutos de gordura na qualidade de pão de queijo. 2000. 82f. Dissertação (Mestrado em Ciência dos Alimentos) - Curso de Pós-graduação em Ciência dos Alimentos, Universidade Federal de Minas Gerais.

DELLA MODESTA, R. C. Manual de análise sensorial de alimentos e bebidas: geral. Rio de Janeiro: EMBRAPACTAA, 1994. 78p.

FIATES, G. M. R. et al. Comportamento consumidor, hábitos alimentares e consumo de televisão por escolares de Florianópolis. Revista de Nutrição, Campinas, v.21, n.1, p.105-114, 2008. Disponível em: $<$ http://www.scielo.br/scielo.php?script=sci_arttext \&pid=S1415-52732008000100011>. Acesso em: 20 dez. 2009. doi: 10.1590/S1415-52732008000100011.

FRANCO, G. Tabela de composição química dos alimentos. São Paulo: Atheneu, 2005. 307p.

LAU, M.H. et al. Kinetics of textural and colour changes in green asparagus during thermal treatments. Journal of Food
Engineering, Oxford, v.45, p.231-236, 2000. Disponível em: $<$ http://www.sciencedirect.com/science?_ob=ArticleListURL \&_method=list\&_ArticleListID $=1444068587$ \&_sort $=\mathrm{r} \& \mathrm{vi}$ e $\mathrm{w}=\mathrm{c} \&$ _ a c c t $=\mathrm{C} 000037521 \&$ \& e r sio $\mathrm{n}=1 \&$ \& urlVersion $=0 \&$ userid $=686380 \& \mathrm{md} 5$ $=\mathrm{c} 0 \mathrm{~b} 607 \mathrm{~d} 404 \mathrm{~b} 75 \mathrm{e} 71 \mathrm{edaa} 52736 \mathrm{~b} 51 \mathrm{e} 570>$. Acesso em: 15 out.2009.

MACHADO, A.V. Efeito do escaldamento nas propriedades tecnológicas da massa e do pão de queijo. 2003. 99f. Dissertação (Mestrado em Ciência dos Alimentos) - Curso de Pós-graduação em Ciência dos Alimentos, Universidade Federal de Lavras.

MINIM, V.P.R. et al. Perfil sensorial e aceitabilidade de diferentes formulações de pão de queijo. Ciência e Tecnologia de Alimentos, Campinas, v.20, n.2, p.154-159, 2000. Disponível em: <http:// www.scielo.br/scielo.php?script $=\mathrm{sci}$ arttext\&pid $=\mathrm{S} 0101$ $20612000000200005 \& \operatorname{lng}=$ pt\&nrm=iso $>$. Acesso em: Acesso em: 20 dez. 2009. doi: 10.1590/S0101-20612000000200005.

PEREIRA, J. et al. Função dos ingredientes na consistência da massa e nas características do pão de queijo. Ciência e Tecnologia de Alimentos, Campinas, v.24, n.4, p.494-500, 2004. Disponível em: $<$ http://www.scielo.br/scielo.php? script=sci arttext\&pid=S010120612004000400003\&lng=pt\&nrm=iso $>$. Acesso em: Acesso em: 20 dez. 2009. doi: 10.1590/S0101-20612004000400003.

RIBEIRO, A.C. et al. Controle microbiológico da vida de prateleira de ricota cremosa. Ciência e Agrotecnologia, Lavras, v.29, n.1, p.113-117, 2005. Disponível em: $<$ http:// www.scielo.br/scielo.php?script $=$ sci_arttext\&pid $=$ S1413$70542005000100014 \&$ lng $=\mathrm{pt} \& \mathrm{nrm}=\mathrm{iso}>$. Acesso em: Acesso em: 20 dez. 2009. doi: 10.1590/S1413-70542005000100014.

TOMICH, R.GP. et al. Metodologia para avaliação das boas práticas de fabricação em indústrias de pão de queijo. Ciência e Tecnologia de Alimentos, Campinas, v.25, n.1, p.115-120, 2005. Disponível em: <http://www.scielo.br/scielo.php?script=sci_arttext \&pid=S010120612005000100019\&lng=pt\&nrm=iso $>$. Acesso em: 15 jan. 2010. doi: $10.1590 / \mathrm{S} 0101-20612005000100019$.

TROCONE, R.; BHATNAGAR, S.; BUTZNER, D.; CAMERON, D.; HILL, I.; HOFFENBERG, E.; MAKI, M.; MENDEZ, V., JIMENEZ, M. Z. Celiac disease and other immunologically mediated disorders of gastrointestinal tract: working group report of the second world congress of pediatric gastroenterology, hepatology and nutrition. Journal of Pediatria Gastroenterology Nutrition, v.39, s.2, p.601610, 2004. Disponível em: <http://www.ncbi.nlm.nih.gov/ pubmed/15184759>. Acesso em: 04 set. 2010. 